
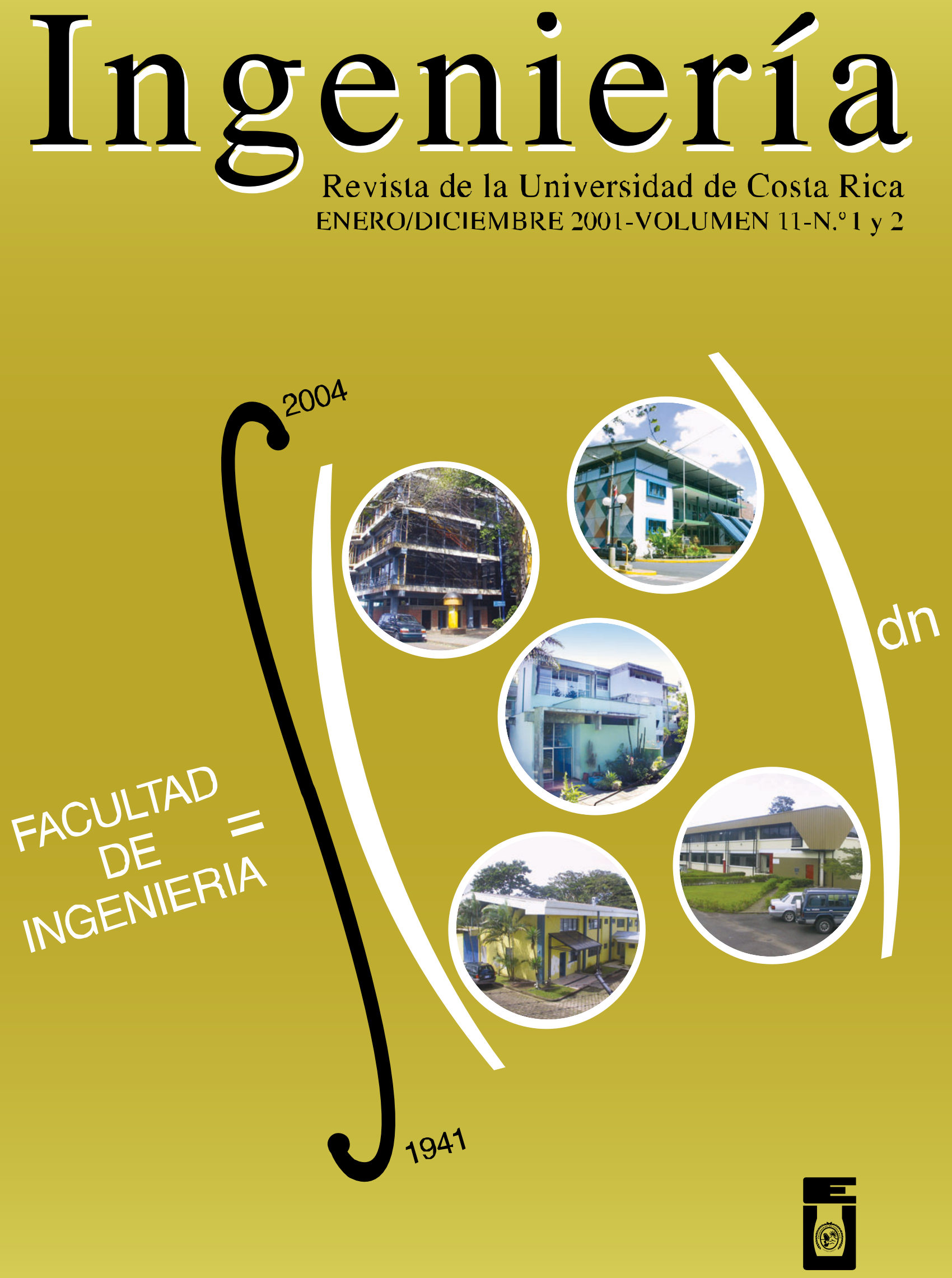


\title{
ANÁLISIS TÉCNICO DEL PAPEL DE 75 g/m² USADO EN COSTA RICA PARA LOS PROCESOS DE IMPRESIÓN LÁSER Y XEROGRÁFICA
}

María Lorena Blanco Rojas 1 Jennifer Ruiz Pacheco ${ }^{2}$

\begin{abstract}
Resumen
Se realiza un análisis técnico del papel de $75 \mathrm{~g} / \mathrm{m}^{2}$, distribuido y utilizado en Costa Rica en los procesos de impresión láser y xerográfica, con base en el estudio de 19 muestras de papel, obtenidas de lotes de más de 1000 resmas que ofrecen proveedores de diversos países.

Se determinaron las propiedades físicas, mecánicas, químicas, superficiales y ópticas mediante los procedimientos estándar, así como los resultados promedio y la desviación estándar, utilizando el proceso estadístico de estimación de intervalos de confianza.

Los resultados obtenidos consisten en el gran promedio, la desviación estándar experimental de la media, la desviación estándar estimada de la población y los límites del intervalo de confianza al $99 \%$ para cada una de las propiedades estudiadas. Estos resultados más la experiencia en el Laboratorio de Productos Forestales (LPF), permite señalar una propuesta para el establecimiento de una norma nacional en la utilización de papel de $75 \mathrm{~g} / \mathrm{m}^{2}$, empleado en los procesos de impresión láser y xerográfica, lo cual contribuye a la normalización nacional del sector papelero. La utilización de la información contenida en este artículo está restringida a los derechos de autor.
\end{abstract}

\begin{abstract}
A technical study of the paper grade 4 of $75 \mathrm{~g} / \mathrm{m}^{2}$ used in process of laser and xerographic print in Costa Rica, is based in the study of 19 paper samples, each one obtained from lots of more than 1000 reams offered by local suppliers and coming from diverse countries in the world.

The physical, mechanical, chemical, superficial and optical properties were determined by means of procedures accepted all over the world. The average of the results together with the standard deviations, were treated using the statistical procedure of estimation of the confidence intervals.

The results consist of the grand average, the experimental mean standard deviation, the population's estimated standard deviation and the limits of interval of trust at $99 \%$ for each of the studied properties. This results, along with the experience obtained in the Laboratorio de Productos Forestales (LPF), permit us to draw a proposal about the future national norm for paper grade 4 of $75 \mathrm{~g} / \mathrm{m}^{2}$ used in process of laser and xerographic print, and in doing so, contribute to the normalization of the national paper sector. Their use is restricted to the author's rights.
\end{abstract}

\section{INTRODUCCIÓN}

El papel xerográfico, conocido también como papel reprográfico o papel para copiado, se define como el grado de papel que se usa para copiado o duplicado por un método xerográfico o electrográfico. Los papeles xerográficos son grados de papeles "bond" hechos con pulpas químicas, mecánicas, papel reciclado, algodón o por combinación de estos elementos.

La xerografía es un método en seco de reproducción de material gráfico, en el cual una imagen se reproduce en forma de carga electroestática por reflexión sobre la superficie de un fotoconductor que sostiene la carga en las zonas oscuras y luego la disipa cuando se expone a la luz. La imagen del fotoconductor se desarrolla cuando se pone en contacto con la tinta en polvo, llamada "toner"; la imagen en polvo es entonces transferida al papel y fijada a este por fusión de calor.

El papel para impresión láser se utiliza en las impresoras láser. Una impresora láser es básicamente una copiadora xerográfica en la cual la porción de la máquina que refleja la luz es remplazada por un láser. De manera similar, este tipo de papel se recomienda para ser utilizado en cualquier impresora electrofotográfica, cuya fuente de luz 
puede ser un diodo (LED), un sistema de cristal líquido (LCS), un diodo láser o cualquier otra fuente de luz controlada (ASTM, 1 996).

En los métodos de duplicación electrofotográfica (xerográfica o láser), el papel debe ser sumamente plano, contar con una rigidez apropiada y buena permeabilidad. Así mismo, presentar una adecuada resistencia electroestática, alta resistencia al calor, ámbitos medios de gramaje, no estar curvado y la superficie debe ser compatible con las partículas de "toner", las cuales se enlazan a la superficie después de fundirse a $204{ }^{\circ} \mathrm{C}$ (Blanco, 1 997).

En la duplicación electrotográfica, normalmente se emplea papel "bond" modificado, hecho de pulpas químicas en un ámbito básico de gramaje que va de 60 a $90 \mathrm{~g} / \mathrm{m} 2$. Se caracteriza por un acabado suave, alta estabilidad térmica y buenas propiedades estéticas como color, brillantez y limpieza.

El abarquillado, deformación que afecta la superficie total de la hoja de papel y hace que tienda a enrollarse sobre sí misma en forma de cilindro, puede afectar significativamente la calidad de las copias obtenidas. Debido a diferencias en la elaboración del papel y a las mismas máquinas copiadoras e impresoras, el lado en que se estampa la imagen sobre el papel puede afectar seriamente el abarquillado. Para obtener los mejores resultados se recomienda consultar con el proveedor de los equipos de reproducción (ASTM, 1 996).

Las copiadoras e impresoras de alta velocidad demandan características especiales en el papel, por lo tanto, es recomendable realizar una prueba inicial de desempeño.

Con respecto al abollado, la deformación localizada en el papel debido a un encogimiento desigual que le confiere un aspecto arrugado típico y otros problemas en la textura, puede interferir en la adherencia del "toner" y consecuentemente en la calidad de la imagen. Igualmente, se recomienda una prueba inicial de desempeño antes de adquirir un lote de papel para un equipo en particular (ASTM, 1 996).

Entre enero y setiembre de 1999 , Costa Rica importó de los Estados Unidos \$42 228,40 en papel para copiadoras e impresoras; entre 1996 a 1998, sólo la Universidad de Costa Rica consumió 95,697 resmas de papel xerográfico.

Algunas especificaciones para las propiedades del papel xerográfico se detallan en el cuadro No. 1. 
Cuadro No. 1. Algunas especificaciones para el papel xerográfico de $75 \mathrm{~g} / \mathrm{m}^{2}$.

\begin{tabular}{|c|c|c|c|}
\hline GRAMAJE NOMINAL & $60 \mathrm{~g} / \mathrm{m}^{2}$ & $75 \mathrm{~g} / \mathrm{m}^{2}$ & $90 \mathrm{~g} / \mathrm{m}^{2}$ \\
\hline Gramaje $\left(\mathrm{g} / \mathrm{m}^{2}\right) \pm 5 \%$ & 60 & 75 & 90 \\
\hline $\begin{array}{l}\text { Espesor promedio }(\mathrm{mm}) \\
\pm 0,013\end{array}$ & 0,018 & 0,102 & 0,122 \\
\hline Humedad (\%) & $3,7-5,7$ & $3,7-5,7$ & $3,7-5,7$ \\
\hline $\begin{array}{l}\text { Lisura Sheffield promedio } \\
\text { para cada lado }\end{array}$ & $200-65$ & $200-65$ & $200-65$ \\
\hline $\begin{array}{ll}\mathrm{pH} & \text { mínimo } \\
(\text { adimensional }) & \end{array}$ & 4,7 & 4,7 & 4,7 \\
\hline $\begin{array}{l}\text { Brillantez (\%) } \\
\text { Grado } 1 \\
\text { Grado } 4 \\
\text { Grado } 5 \\
\end{array}$ & $\begin{array}{l}85 \\
79 \\
74 \\
\end{array}$ & $\begin{array}{l}85 \\
79 \\
74\end{array}$ & $\begin{array}{l}85 \\
79 \\
74\end{array}$ \\
\hline $\begin{array}{l}\text { Opacidad (\%) } \\
\text { Grado } 1 \\
\text { Grado } 4,5, \mathrm{~N}\end{array}$ & $\begin{array}{l}-- \\
79\end{array}$ & $\begin{array}{l}85 \\
84\end{array}$ & 86 \\
\hline DIMENSIONES & & & \\
\hline Largo $\pm 0,8 \mathrm{~mm}$ & $\begin{array}{c}8.5 \times 11 \\
(216 \times \\
279 \mathrm{~mm})\end{array}$ & $\begin{array}{c}8.5 \times 11 \\
(216 \times \\
279 \mathrm{~mm})\end{array}$ & $\begin{array}{c}8.5 \times 11 \\
(216 \times \\
279 \mathrm{~mm})\end{array}$ \\
\hline Ancho $\pm 0,8 \mathrm{~mm}$ & $\begin{array}{c}8.5 \times 14 \\
(216 \times \\
356 \mathrm{~mm}) \\
11 \times 17 \\
(279 \times 432 \\
\mathrm{mm})\end{array}$ & $\begin{array}{c}8.5 \times 14 \\
(216 \times \\
356 \mathrm{~mm}) \\
11 \times 17 \\
(279 \times 432 \\
\mathrm{mm})\end{array}$ & $\begin{array}{c}8.5 \times 14 \\
(216 \times \\
356 \mathrm{~mm}) \\
11 \times 17 \\
(279 \times 432 \\
\mathrm{mm})\end{array}$ \\
\hline
\end{tabular}




\section{METODOLOGÍA EXPERIMENTAL}

Se utilizaron diez muestras de papel xerográfico de $75 \mathrm{~g} / \mathrm{m}^{2}$ grado 4 , tomadas en forma aleatoria de muestreos aleatorios realizados en lotes de más de 1000 resmas cada uno, provenientes de diez proveedores distintos.

Además, se utilizaron los resultados históricos de nueve ofertas y ensayos, realizados por el Laboratorio de Productos Forestales (LPF) entre los años 1996 y 1997. En vista de que estos resultados corresponden a investigaciones anteriores del Laboratorio, no presentan resultados en todas las propiedades estudiadas en este informe.

Las muestras se obtuvieron al aplicar el II Plan de muestreo descrito en T-400 y los ensayos se efectuaron siguiendo los procedimientos descritos en TAPPI (1 996).

Por consiguiente, se realizaron los siguientes ensayos: gramaje (T410); humedad (T412); espesor (T411); pH con extracción en caliente (T435); resistencia a la explosión (T403); resistencia a la tensión (TAPPI T404); opacidad tanto en fondo blanco como en fondo papel (T425); brillantez (T452) y resistencia al rasgado (T414).

Para los ensayos de lisura y de permeabilidad al aire -Gurley-, se siguieron los procedimientos descritos por Blanco (1 995). Todos los especímenes del ensayo fueron acondicionados a una atmósfera controlada por una humedad relativa de $50 \% \pm 2 \%$ y una temperatura de $23{ }^{\circ} \mathrm{C} \pm 2$ ${ }^{\circ} \mathrm{C}$, de acuerdo con (T402).

La validación de los resultados se sustenta en un programa de calibración continua y de aseguramiento de la calidad con que cuenta el LPF. Los equipos están certificados por el Laboratorio de Control de Calidad, Normalización y Metrología (LABCAL) y por los resultados obtenidos a lo largo de tres años de participar en un programa interlaboratorial desarrollado por el Instituto de Pesquisas do Estado de São Paulo (IPT) del Brasil, en el cual participan más de 60 laboratorios; por lo tanto, los equipos utilizados en este estudio poseen confirmación metrológica.
Cada una de las diecinueve muestras proporciona un resultado promedio que se obtiene de tres repeticiones en el caso de propiedades químicas y gramaje, y de diez repeticiones para las propiedades físicas, mecánicas, ópticas y superficiales.

La información de las pruebas de laboratorio proporciona un valor para cada propiedad, que resulta del promedio de por lo menos tres repeticiones de la medición. Este se denomina media de cada muestra de propiedad para cada oferta y se calcula usando la siguiente ecuación:

$$
\bar{X}=\frac{\sum_{i=1}^{n} X_{i}}{n}
$$

donde $\mathrm{n}$ representa el número de repeticiones.

La desviación estándar experimental o muestral se calcula a partir de la mediciones experimentales como una variación entre muestras, con la siguiente ecuación:

$$
S(X)=\sqrt{\frac{\sum_{i=1}^{n}\left(X-X_{i}\right)^{2}}{n-1}}
$$

A partir de lo anterior, se puede establecer una desviación global de las propiedades del papel sujetas a medición que se calcula usando la ecuación siguiente y considerando todas las ofertas que fueron analizadas en el laboratorio:

$$
S(X)=\sqrt{\frac{\left(k_{1}-1\right) S_{1}^{2}+\left(k_{2}-1\right) S_{2}^{2}+\ldots+\left(k_{n}-1\right) S_{n}^{2}}{\sum_{i=1}^{n}(k-1)}}
$$

Con los promedios experimentales de las diferentes ofertas se calcula un gran promedio para cada propiedad, el cual representa el valor de la propiedad que tiene el papel que se vende en el país. Para calcular las medias de la población se utilizó en todos los casos la siguiente ecuación:

$$
\mu=\frac{\sum_{i=1}^{n} \overline{X_{e}}}{n}
$$

donde $\mathrm{n}$ se refiere al número de ofertas. 
La desviación estándar de las ofertas de la población se interpreta como la variación que existe del mismo tipo de papel en una propiedad particular, como por ejemplo el espesor o la permeabilidad. A partir de los valores promedio, para cada propiedad, de todas las ofertas disponibles, se calcula la desviación, tal como sugiere Bader (Junio, 1980 ), usando la ecuación:

$$
\sigma(\bar{X})=\sqrt{\frac{\sum_{i=1}^{n}\left(\mu-\overline{X_{i}}\right)^{2}}{n}}
$$

donde $\mathrm{n}$ se refiere al número de ofertas.

Para calcular los valores extremos de los intervalos de confianza se utilizó la definición que establece Miller (1 993), por lo tanto:

$\mu(\bar{X})-z_{\alpha / 2} \frac{\sigma(\bar{X})}{\sqrt{n}} \leq \mu \leq \mu(\bar{X})+z_{\alpha / 2} \frac{\sigma}{\sqrt{n}}$

Se utiliza como referencia la curva de distribución normal, porque en todos los casos se emplean al menos 40 datos experimentales para ca- da propiedad. De los promedios experimentales de las diecinueve muestras, se calcula un gran promedio y una desviación estándar de la población. A partir de estos datos y utilizando la curva de probabilidad normal, se estiman los intervalos para un $99 \%$ de confianza, con el fin de darle mayor amplitud al intervalo, procurando que éste se adecue lo más posible a los resultados prácticos.

\section{ANÁLISIS Y DISCUSIÓN DE RESULTADOS}

Los resultados de los ensayos y la información adicional recopilada en el laboratorio, se muestran en el cuadro 2, 3, 4 y 5, donde " $x$ " corresponde al valor promedio de la propiedad y "s" a la desviación estándar.

En el cuadro No. 6 se presenta el gran promedio y la desviación estándar de la población para cada una de las propiedades ensayadas, más los límites inferior y superior de los intervalos para el $99 \%$ de confianza y la desviación estándar experimental de la media. 
Cuadro No 2. Propiedades físicas, químicas y superficiales obtenidas para papel xerográfico $75 \mathrm{~g} / \mathrm{m}^{2}$

\begin{tabular}{|c|c|c|c|c|c|c|c|c|c|c|c|}
\hline \multirow{2}{*}{ PROPIEDAD } & & \multicolumn{10}{|c|}{ MUESTRAS } \\
\hline & & 1 & 2 & 3 & 4 & 5 & 6 & 7 & 8 & 9 & 10 \\
\hline \multirow{2}{*}{$\begin{array}{l}\text { Gramaje } \\
\left(\mathrm{g} / \mathrm{m}^{2}\right)\end{array}$} & $\mathrm{x}$ & 75.9 & 76.9 & 77.9 & 77.9 & 75.3 & 74.9 & 75.9 & 76.7 & 76.9 & 78.1 \\
\hline & $\mathrm{s}$ & 0.6 & 0.3 & 0.3 & 0.4 & 0.2 & 0.7 & 0.3 & 0.2 & 0.2 & 0.2 \\
\hline \multirow{2}{*}{$\begin{array}{l}\text { Humedad } \\
(\%)\end{array}$} & $\mathrm{x}$ & 5.42 & 6.85 & 6.77 & 5.32 & 5.81 & 5.61 & 6.42 & 5.01 & 5.47 & 6.31 \\
\hline & $\mathrm{s}$ & 0.06 & 0.15 & 0.15 & 0.08 & 0.10 & 0.10 & 0.10 & 0.20 & 0.07 & 0.20 \\
\hline \multirow{2}{*}{$\begin{array}{l}\text { Espesor } \\
\text { promedio } \\
(\mu \mathrm{m})\end{array}$} & $\mathrm{x}$ & 101 & 102 & 99 & 102 & 98 & 96 & 96 & 101 & 95 & 96 \\
\hline & $\mathrm{s}$ & 2 & 3 & 0 & 3 & 1 & 3 & 3 & 2 & 4 & 3 \\
\hline \multirow{2}{*}{$\begin{array}{l}\text { Densidad } \\
\text { aparente } \\
\left(\mathrm{kg} / \mathrm{m}^{3}\right)\end{array}$} & $\mathrm{x}$ & 759 & 765 & 770 & 765 & 768 & 765 & 770 & 813 & 739 & 811 \\
\hline & S & 3 & 3 & 16 & 3 & 6 & 3 & 16 & -- & 23 & 49 \\
\hline \multirow{2}{*}{$\begin{array}{l}\text { Permeabilida } \\
\mathrm{d}(\mathrm{s} / 100 \mathrm{ml})\end{array}$} & $\mathrm{x}$ & 9.2 & 9.0 & 16.4 & 25.5 & 7.7 & 9.4 & 15.4 & 11.1 & 16.1 & 11.0 \\
\hline & $\mathrm{s}$ & 1.0 & 0.3 & 2.0 & 1.4 & 0.1 & 0.1 & 2.0 & 0.1 & 0.2 & 1.0 \\
\hline \multirow{2}{*}{$\begin{array}{l}\text { Lisura } \\
(\mathrm{s} / 50 \mathrm{ml})\end{array}$} & $\mathrm{X}$ & 65 & 41 & 40 & 38 & 48 & 86 & 44 & 75 & 37 & 56 \\
\hline & $\mathrm{S}$ & 7 & 4 & 7 & 5 & 7 & 6 & 6 & 7 & 1 & 18 \\
\hline \multirow[t]{2}{*}{ pH (adim.) } & $\mathrm{X}$ & 10.08 & 9.86 & 5.59 & 9.63 & 9.77 & 9.79 & 5.84 & 6.05 & 9.36 & 9.89 \\
\hline & $\mathrm{s}$ & 0.03 & 0.02 & 0.03 & 0.06 & 0.04 & 0.00 & 0.01 & 0.01 & 0.63 & 0.00 \\
\hline \multirow{2}{*}{$\begin{array}{l}\text { Resistencia a } \\
\text { la explosión } \\
(\mathrm{kPa})\end{array}$} & $\mathrm{x}$ & 216 & 123 & 82 & 169 & 130 & 204 & 116 & 258 & 133 & 140 \\
\hline & $\mathrm{s}$ & 18 & 12 & 11 & 13 & 11 & 16 & 15 & 13 & 13 & 10 \\
\hline
\end{tabular}


Cuadro No 3. Propiedades físicas, químicas y superficiales obtenidas para papel xerográfico $75 \mathrm{~g} / \mathrm{m}^{2}$

\begin{tabular}{|c|c|c|c|c|c|c|c|c|c|c|}
\hline \multirow[t]{2}{*}{ PROPIEDAD } & & \multicolumn{9}{|c|}{ MUESTRAS } \\
\hline & & 11 & 12 & 13 & 14 & 15 & 16 & 17 & 18 & 19 \\
\hline \multirow[t]{2}{*}{ Gramaje $\left(\mathrm{g} / \mathrm{m}^{2}\right)$} & $\mathrm{x}$ & 75.4 & 76.2 & 77.5 & 76.1 & 77.1 & 77.3 & 75.9 & 77.5 & 75.3 \\
\hline & $\mathrm{s}$ & 1.2 & 0.1 & 0.2 & 0.2 & 0.2 & 0.1 & 0.2 & 0.1 & 0.8 \\
\hline \multirow[t]{2}{*}{ Humedad (\%) } & $\mathrm{x}$ & 5.60 & 5.42 & 5.90 & 4.90 & 5.44 & 5.39 & 4.87 & 5.15 & 4.50 \\
\hline & $\mathrm{s}$ & 0.32 & 0.03 & 0.07 & 0.15 & 0.06 & 0.10 & 0.10 & 0.05 & 0.18 \\
\hline \multirow{2}{*}{$\begin{array}{l}\text { Espesor } \\
\text { promedio }(\mu \mathrm{m})\end{array}$} & $\mathrm{x}$ & 102 & 98 & 94 & 104 & 96 & 99 & 101 & 102 & 98 \\
\hline & s & 3 & 1 & 5 & 5 & 3 & 0 & 2 & 3 & 1 \\
\hline \multirow{2}{*}{$\begin{array}{l}\text { Densidad } \\
\text { aparente }\left(\mathrm{kg} / \mathrm{m}^{3}\right)\end{array}$} & $\mathrm{x}$ & 783 & 676 & 652 & 739 & 760 & 793 & -- & -- & -- \\
\hline & $\mathrm{s}$ & 21 & 86 & 110 & 31 & 2 & 31 & -- & -- & -- \\
\hline \multirow{2}{*}{$\begin{array}{l}\text { Permeabilidad } \\
(\mathrm{s} / 100 \mathrm{ml})\end{array}$} & $\mathrm{x}$ & 19.0 & 24.0 & -- & -- & -- & -- & -- & -- & -- \\
\hline & $\mathrm{s}$ & 3.4 & 2.6 & -- & -- & -- & -- & -- & -- & -- \\
\hline \multirow[t]{2}{*}{ Lisura (s/50ml) } & $\mathrm{x}$ & -- & -- & -- & -- & -- & -- & -- & -- & -- \\
\hline & $\mathrm{s}$ & -- & -- & -- & -- & -- & -- & -- & -- & -- \\
\hline \multirow[t]{2}{*}{ pH (adim.) } & $\mathrm{x}$ & 9.75 & 5.80 & 10.0 & 9.58 & 9.70 & 9.81 & 5.58 & 9.56 & 9.62 \\
\hline & $\mathrm{s}$ & 0.02 & 0.10 & 0.10 & 0.16 & 0.05 & 0.04 & 0.20 & 0.01 & 0.01 \\
\hline \multirow{2}{*}{$\begin{array}{l}\text { Resistencia a la } \\
\text { explosión }(\mathrm{kPa})\end{array}$} & $\mathrm{x}$ & 182 & -- & -- & -- & -- & -- & -- & -- & -- \\
\hline & $\mathrm{s}$ & 12 & -- & -- & -- & -- & -- & -- & -- & -- \\
\hline
\end{tabular}


Cuadro No 4. Propiedades mecánicas y ópitcas obtenidas para papel xerográfico $75 \mathrm{~g} / \mathrm{m}^{2}$

\begin{tabular}{|c|c|c|c|c|c|c|c|c|c|c|c|}
\hline \multirow[t]{2}{*}{ PROPIEDAD } & \multicolumn{11}{|c|}{ MUESTRA } \\
\hline & & 1 & 2 & 3 & 4 & 5 & 6 & 7 & 8 & 9 & 10 \\
\hline \multirow{2}{*}{$\begin{array}{l}\text { Tensión } \\
\text { longitudinal } \\
(\mathrm{kN} / \mathrm{m})\end{array}$} & $\mathrm{x}$ & 5.34 & 4.46 & 3.57 & 3.50 & 3.92 & 5.30 & 3.40 & 5.91 & 3.34 & 3.37 \\
\hline & $\mathrm{s}$ & 0.15 & 0.15 & 0.15 & 0.30 & 0.10 & 0.30 & 0.10 & 0.17 & 0.09 & 0.15 \\
\hline \multirow{2}{*}{$\begin{array}{l}\text { Tensión } \\
\text { transversal } \\
(\mathrm{kN} / \mathrm{m})\end{array}$} & $\mathrm{x}$ & 2.03 & 2.15 & 2.00 & 2.48 & 1.73 & 2.80 & 1.88 & 2.68 & 2.53 & 2.43 \\
\hline & $\mathrm{s}$ & 0.05 & 0.05 & 0.07 & 0.09 & 0.06 & 0.07 & 0.07 & 0.12 & 0.16 & 0.06 \\
\hline \multirow{2}{*}{$\begin{array}{l}\text { Resistencia al } \\
\text { rasgado } \\
\text { longitudinal } \\
(\mathrm{mN})\end{array}$} & $\mathrm{x}$ & 623 & 607 & 741 & 534 & 588 & 541 & 925 & 493 & 491 & 439 \\
\hline & $\mathrm{s}$ & 30 & 24 & 36 & 31 & 32 & 31 & 124 & 23 & 14 & 14 \\
\hline \multirow{2}{*}{$\begin{array}{l}\text { Resistencia al } \\
\text { rasgado } \\
\text { transversal } \\
(\mathrm{mN})\end{array}$} & $\mathrm{x}$ & 723 & 687 & 352 & 473 & 761 & 578 & 703 & 568 & 505 & 470 \\
\hline & $\mathrm{s}$ & 23 & 41 & 32 & 25 & 40 & 24 & 47 & 33 & 21 & 41 \\
\hline \multirow{2}{*}{$\begin{array}{l}\text { Opacidad fondo } \\
\text { blanco }(\%)\end{array}$} & $\mathrm{x}$ & 87.4 & 89.0 & 88.8 & 86.4 & 90.5 & 90.6 & 87.5 & 86.9 & 87.4 & 88.0 \\
\hline & $\mathrm{s}$ & 2.0 & 0.3 & 1.0 & 1.6 & 1.8 & 0.5 & 2.0 & 1.4 & 2.0 & 1.8 \\
\hline \multirow{2}{*}{$\begin{array}{l}\text { Opacidad fondo } \\
\text { papel }(\%)\end{array}$} & $\mathrm{x}$ & 87.9 & 87.6 & 88.5 & 87.7 & 91.7 & 89.4 & 88.7 & 88.0 & 88.6 & 89.3 \\
\hline & $\mathrm{s}$ & 0.2 & 1.8 & 1.2 & 1.0 & 1.2 & 1.0 & 1.3 & 0.1 & 1.2 & 1.1 \\
\hline \multirow[t]{2}{*}{ Brillantez (\%) } & $\mathrm{x}$ & 83.9 & 83.2 & 83.1 & 79.0 & 82.0 & 87.7 & 83.0 & 84.0 & 78.0 & 81.0 \\
\hline & $\mathrm{s}$ & 0.1 & 0.1 & 0.1 & 0.0 & 0.0 & 0.5 & 0.0 & 0.0 & 0.0 & 0.0 \\
\hline
\end{tabular}


Cuadro No 5. Propiedades mecánicas y ópitcas obtenidas para papel xerográfico $75 \mathrm{~g} / \mathrm{m}^{2}$

\begin{tabular}{|c|c|c|c|c|c|c|c|c|c|c|}
\hline \multirow[t]{2}{*}{ PROPIEDAD } & \multicolumn{10}{|c|}{ MUESTRA } \\
\hline & & 11 & 12 & 13 & 14 & 15 & 16 & 17 & 18 & 19 \\
\hline \multirow{2}{*}{$\begin{array}{l}\text { Tensión } \\
\text { longitudinal } \\
(\mathrm{kN} / \mathrm{m})\end{array}$} & $\mathrm{x}$ & 2.10 & 8.56 & 6.07 & -- & -- & -- & -- & -- & -- \\
\hline & $\mathrm{s}$ & 0.12 & 0.70 & 0.41 & -- & -- & -- & -- & -- & -- \\
\hline \multirow{2}{*}{$\begin{array}{l}\text { Tensión } \\
\text { transversal }(\mathrm{kN} / \mathrm{m})\end{array}$} & $\mathrm{x}$ & 5.35 & 5.09 & 3.77 & -- & -- & -- & -- & -- & -- \\
\hline & $\mathrm{s}$ & 0.40 & 0.20 & 0.25 & -- & -- & -- & -- & -- & -- \\
\hline \multirow{2}{*}{$\begin{array}{l}\text { Resistencia al } \\
\text { rasgado } \\
\text { longitudinal ( } \mathrm{mN})\end{array}$} & $\mathrm{x}$ & 608 & -- & -- & -- & -- & -- & 420 & 322 & 471 \\
\hline & $\mathrm{s}$ & 38 & -- & -- & -- & -- & -- & 18 & 17 & 35 \\
\hline \multirow{2}{*}{$\begin{array}{l}\text { Resistencia al } \\
\text { rasgado transversal } \\
(\mathrm{mN})\end{array}$} & $\mathrm{x}$ & 550 & -- & -- & -- & 375 & 374 & 389 & 434 & 354 \\
\hline & $\mathrm{s}$ & 24 & -- & -- & -- & 12 & 35 & 12 & 12 & 28 \\
\hline \multirow{2}{*}{$\begin{array}{l}\text { Opacidad fondo } \\
\text { blanco }(\%)\end{array}$} & $\mathrm{x}$ & 89.9 & 91.0 & 88.0 & 87.0 & 88.0 & 87.0 & -- & -- & -- \\
\hline & $\mathrm{s}$ & 1.7 & 0.7 & 1.2 & 1.3 & 1.2 & 1.3 & -- & -- & -- \\
\hline \multirow{2}{*}{$\begin{array}{l}\text { Opacidad fondo } \\
\text { papel }(\%)\end{array}$} & $\mathrm{x}$ & -- & -- & -- & -- & -- & -- & -- & -- & -- \\
\hline & $\mathrm{s}$ & -- & -- & -- & -- & -- & -- & -- & -- & -- \\
\hline \multirow[t]{2}{*}{ Brillantez (\%) } & $\mathrm{x}$ & 81.6 & 80.1 & 80.0 & 82.0 & 82.0 & 81.0 & 81.9 & 80.0 & 79.6 \\
\hline & $\mathrm{s}$ & 0.1 & 0.1 & 0.1 & 0.2 & 0.8 & 0.0 & 0.1 & 0.0 & 0.5 \\
\hline
\end{tabular}


Cuadro No. 6. Especificaciones sugeridas para el papel xerográfico y de impresora láser de $75 \mathrm{~g} / \mathrm{m}^{2}$, distribuido en Costa Rica a $50 \% \pm 2 \%$ HR y $23^{\circ} \mathrm{C} \pm 2{ }^{\circ} \mathrm{C}$

\begin{tabular}{|c|c|c|c|c|c|}
\hline PROPIEDAD & $\begin{array}{c}\text { Promedio } \\
\mu\end{array}$ & $\begin{array}{l}\text { Desviación } \\
\text { estándar } \\
\sigma\end{array}$ & $\begin{array}{l}\text { Valor } \\
\text { mínimo }\end{array}$ & $\begin{array}{l}\text { Valor } \\
\text { máximo }\end{array}$ & Especificación sugerida \\
\hline \multicolumn{6}{|c|}{ PROPIEDADES FÍSICAS } \\
\hline $\begin{array}{l}\text { Gramaje en base } \\
\text { húmeda }\left(\mathrm{g} / \mathrm{m}^{2}\right)\end{array}$ & 76,6 & 1,0 & 75,1 & 78,1 & 71,3 a 78,7 \\
\hline $\begin{array}{l}\text { Humedad en base } \\
\text { húmeda }(\%)\end{array}$ & 5,59 & 0,63 & 4,65 & 6,53 & 4,0 a 6,0 \\
\hline $\begin{array}{l}\text { Espesor promedio } \\
(\mu \mathrm{m})\end{array}$ & 99 & 3 & 97 & 101 & 90 a 110 \\
\hline $\begin{array}{l}\text { Densidad aparente } \\
\left(\mathrm{kg} / \mathrm{m}^{3}\right)\end{array}$ & 761 & 43 & 733 & 790 & -- \\
\hline $\begin{array}{l}\text { Permeabilidad al aire } \\
\text { Gurley }(\mathrm{s} / 100 \mathrm{ml})\end{array}$ & 14.5 & 6,0 & 9,6 & 19,4 & -- \\
\hline \multicolumn{6}{|c|}{ PROPIEDADES SUPERFICIALES } \\
\hline $\begin{array}{l}\text { Lisura Gurley } \\
(\mathrm{s} / 50 \mathrm{ml})\end{array}$ & 53 & 18 & 38 & 67 & 40 a 70 \\
\hline \multicolumn{6}{|c|}{ PROPIEDADES QUÍMICAS } \\
\hline $\mathrm{pH}$ (adimensional) & 8,70 & 1,81 & 5,42 & 12,00 & Mínimo 6,0 \\
\hline \multicolumn{6}{|c|}{ PROPIEDADES MECÁNICAS } \\
\hline $\begin{array}{l}\text { Resistencia a la } \\
\text { explosión }(\mathrm{kPa})\end{array}$ & 160 & 52 & 118 & 202 & Mínimo 120 \\
\hline $\begin{array}{l}\text { Resistencia a la } \\
\text { tensión longitudinal } \\
(\mathrm{kN} / \mathrm{m})\end{array}$ & 4,52 & 1,70 & 3,08 & 5,96 & Mínimo 4,00 \\
\hline $\begin{array}{l}\text { Resistencia a la } \\
\text { tensión transversal } \\
(\mathrm{kN} / \mathrm{m})\end{array}$ & 2,82 & 1,18 & 1,86 & 3,78 & Mínimo 2,00 \\
\hline $\begin{array}{l}\text { Resistencia al } \\
\text { rasgado longitudinal } \\
(\mathrm{mN})\end{array}$ & 557 & 148 & 431 & 684 & Mínimo 400 \\
\hline $\begin{array}{l}\text { Resistencia al } \\
\text { rasgado transversal } \\
(\mathrm{mN})\end{array}$ & 521 & 136 & 411 & 632 & Mínimo 400 \\
\hline \multicolumn{6}{|c|}{ PROPIEDADES ÓPTICAS } \\
\hline $\begin{array}{l}\text { Opacidad sobre } \\
\text { fondo blanco }(\%)\end{array}$ & 88,4 & 1,5 & 87,2 & 89,6 & Mínimo 85 \\
\hline $\begin{array}{l}\text { Opacidad sobre } \\
\text { fondo papel }(\%)\end{array}$ & 88,7 & 1,2 & 87,7 & 89,7 & Mínimo 85 \\
\hline Brillantez (\%) & 81,7 & 2,2 & 79,9 & 83,5 & Mínimo 80 \\
\hline
\end{tabular}




\section{PROPUESTA}

\section{REQUISITOS GENERALES}

- El papel debe poseer propiedades adecuadas para la impresión: facilidad de borrado, blancura, limpieza, acabado, textura, espesor y formación uniforme.

- El papel debe estar libre de manchas, polvo, pelusas, suciedad, arrugas, dobleces y otras malformaciones o partículas.

- Debe poseer bordes limpios y lisos, libres de marcas de cuchillas; debe estar plano, sin ondas ni orillas levantadas y las esquinas en ángulo recto

- El sentido de fabricación debe ser especificado por el comprador, para ello se suele cortar el papel por el lado más largo en el sentido de la fibra.

- Especificarse si el color es blanco o algún otro tono en particular.

- El empaque debe realizarse con materiales resistentes a la absorción de la humedad, a menos que se especifique otra cosa. En el caso de resmas, éstas deben contener en promedio 500 hojas o pliegos.

- Cada empaque debe indicar externamente el tipo, grado, dimensión, color, dirección de la fibra y gramaje. Las dimensiones y el gramaje deben adecuarse a las unidades del Sistema Internacional de Medidas.

- Cuando se inspecciona un lote en el lugar de la entrega, el muestreo se efectúa siguiendo el Plan II de la Norma T 400 (TAPPI, 1 996), y los análisis de acuerdo con los métodos listados en las tablas No. 1, 2 y 3 del mismo plan.

- En el caso de locales de impresión sin aire acondicionado, la humedad del papel debe encontrarse en equilibrio con las condiciones de temperatura y humedad relativa.
Requisitos técnicos medidas a $50 \% \pm 2 \%$ de humedad relativa y $23{ }^{\circ} \mathrm{C} \pm 2{ }^{\circ} \mathrm{C}$ :

- Gramaje nominal de $75 \mathrm{~g} / \mathrm{m}^{2}$ con una tolerancia real de $+5 \%$.

- Humedad entre 4,0 \% y 6,0\%.

- Espesor nominal de $100 \mathrm{~mm}$ y tolerancia de + $10 \mu \mathrm{m}$.

- Lisura Gurley entre $40 \mathrm{~s} / 50 \mathrm{ml}$ y $70 \mathrm{~s} / 50 \mathrm{ml}$.

- pH mínimo de 6,0 en el extracto en agua caliente del papel.

- Las resistencias se ajustan a la tensión longitudinal mínima de $4 \mathrm{kN} / \mathrm{m}$, la explosión mínima de $120 \mathrm{kPa}$ y al rasgado mínimo, en ambas direcciones, de $400 \mathrm{mN}$.

- Brillantez mínima de $80 \%$ y opacidad mínima de $85 \%$

No se especifican la densidad aparente ni la permeabilidad al aire.

\section{NOMENCLATURA}

$\begin{array}{cl}\mathrm{n} & \text { Valor enésimo de una pror } \\ \mathrm{X} & \text { Representa el valor de una } \\ \mathrm{X} & \text { Valor promedio de una prc } \\ \mathrm{Z} & \text { Valor de normalidad }\end{array}$

$\alpha$

$\mu \quad$ Gran promedio o promedic

\section{BIBLIOGRAFÍA}

1. ASENJO, P. L.; BARBADILLO, P.; MONFORT, P.G. Diccionario terminológico iberoamericano de celulosa, papel y cartón y sus derivados. Instituto Papelero Español, 1992.

2. ASTM. Standard specification for white watermarked and unwatermarked bond, mimeo, spirit duplicator, xerographic, and 
laser printer cut-sized office papers. In: 1 996 Annual Book of ASTM Standards, Philadelphia, Vol. 15.09, p.405-408.

3. BADER, M.E. Quality assurence and quality control. Part I: Specifications In: Chemical Engeneering, February 11, 1 980, p. 87-92.

4. BADER, M.E. Quality assurence and quality control. Part II: The quality-control laboratory. In: Chemical Engeneering, April 7, 1 980, p. 89-93.

5. BADER, M.E. Quality assurence and quality control. Part III: Using statistics. In: Chemical Engeneering, June 16, 1 980, p. 123-129.

6. BADER, M.E. Quality assurence and quality control. Part IV: Complying with goverment regulations. In: Chemical Engeneering, August 25, 1 980, p. 95-97.

7. BLANCO, M. L. Manual de instrucciones del densímetro Gurley. Modelo PGH-T,
Permeabilidad, lisura y suavidad en papel y cartón. (TRADUCCIÓN). INII-106-95, Laboratorio de Productos Forestales, Instituto de Investigaciones en Ingeniería, Universidad de Costa Rica,1 995.

8. BLANCO, M. L. Manual de instrucciones del Medidor de Cobb. Absorción de agua en papel y cartón. (TRADUCCIÓN). INII-10795. Laboratorio de Productos Forestales, Instituto de Investigaciones en Ingeniería, Universidad de Costa Rica, 1995.

9. BLANCO, M. L. Medidor de espesor (ESP/DM) para papel y cartón. (TRADUCCIÓN). INII-108-95, Laboratorio de Productos Forestales, Instituto de Investigaciones en Ingeniería, Universidad de Costa Rica, 1996.

10. KOURIS, M. (EDITOR). Dictionary of paper. Tappi Press, Atlanta, 1 996. 347p.

11. SCOTT, W. E.; ABBOTT, J. C. TROSSET, S. Properties of paper: an introduction. Tappi Press, Atlanta, 1 989. 191p. 